\title{
LONG TIME BEHAVIOUR FOR A CHAIN OF COLLIDING ROTATORS
}

\author{
C. TRESSER \\ Laboratoire de Physique de la Matière Condensée (*) \\ Parc Valrose, 06034 Nice Cedex, France
}

(Reçu le 13 mars 1978, révisé le 24 avril 1978, accepté le 16 mai 1978)

\begin{abstract}
Résumé. - Nous construisons un modèle simple de cristal plastique pour étudier les effets à longs temps du mécanisme d'engrenage. Nous présentons des arguments qui nous mènent à conjecturer qu'une distribution initiale des vitesses angulaires des rotateurs diffuse suivant une loi normale, ce qui donne une décroissance en $t^{-1 / 2}$ de la fonction d'autocorrelation des vitesses angulaires des rotateurs. Ceci a été confirmé par un calcul de dynamique moléculaire numérique.
\end{abstract}

Abstract. - A simple model of a plastic crystal is constructed to study the long term effects of a cog-wheel mechanism. We present some arguments which lead us to conjecture that an initial distribution of rotators' angular velocity diffuses according to a normal law with a $t^{-1 / 2}$ decay of the angular velocity autocorrelation function of the rotators. This has been confirmed by a molecular dynamic calculation performed on a chain of 1000 rotators with periodic boundary conditions.

Recent molecular dynamic studies on two dimensional fluid systems of diatomics [1] have shown at the longest calculated time a $t^{-3}$ decay of the angular velocity autocorrelation function $F_{\omega}(t)$. The asymptotic $t^{-2}$ predicted by hydrodynamics [2] can occur at longer times with very small amplitude, and it was argued in [1] that the observed $t^{-3}$ was possibly due to the transfer of the intrinsic angular momentum by some kind of cog-wheel mechanism. Here, an attempt to understand the long-term effect of cogwhell coupling in one dimension is reported. Further work [3] will be devoted to two dimensional systems.

To isolate the cog-wheel coupling, we shall consider a line of colliding rotators with fixed centres. In the simplest case i) no multiple collisions occur and, ii) a couple $\left(\omega_{1}, \omega_{2}\right)$ of angular velocities transform to $\left(-\omega_{2},-\omega_{1}\right)$ when molecules 1 and 2 collide. This corresponds to two adjacent sectors of angle less than $\pi$ interacting as soon as they touch each other (Fig. 1). The exchange of angular momentum is then instantaneous and the two sectors have the new momenta until the next contact.

This model is inspired by the one-dimensional system of hard rods solved by Jepsen and LebowitzPercus [4]. In both, the dynamics consist of neighbouring particles interchanging velocities at each collision. This forbids ergodicity and relaxation towards an equilibrium.

(*) Laboratoire associé au CNRS no 190.

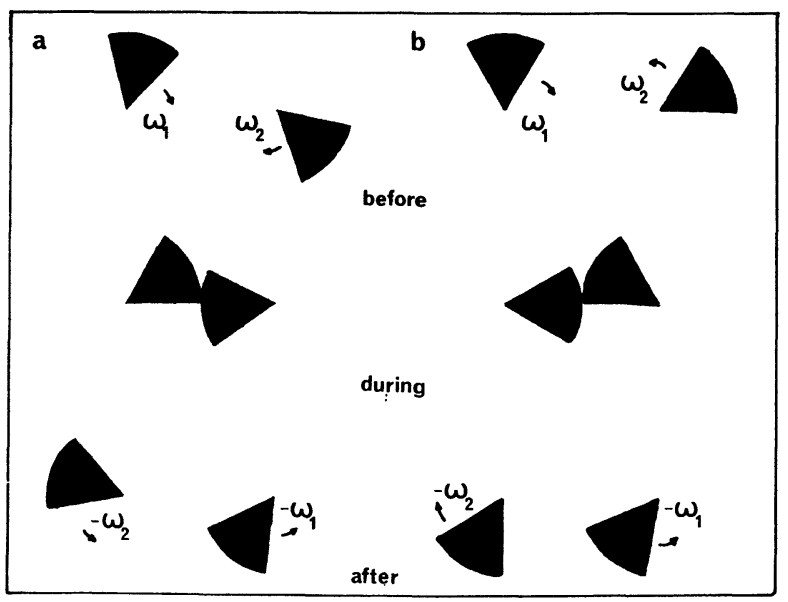

FIG. 1. - Collision (a) corresponds to $\omega_{1} \omega_{2} \geqslant 0$ and is compatible with a rough disk model. Collision (b) corresponds to $\omega_{1} \omega_{2} \leqslant 0$ and can only take place by virtue of the rules defined in the main text.

A Maxwellian distribution of angular velocities was initially imposed, the phase of the rotators being randomly chosen with a uniform distribution in the interval $[0,2 \pi[$. In each collision, the norms of the angular velocities are exchanged while the signs are inverted. Therefore, the initial distribution of angular velocities is not maintained. However, the numerical results show that this distribution is maintained on the average. The complexity of predicting the parameters of a binary collision, and the dependence of the 
dynamics on the angle defining the shape of the rotators make a complete analytical treatment of our model unlikely. Paradoxically, its dynamical behaviour appears more intuitive than for the hard rod model. Indeed, in contradiction with the hard rod case, the sign of the angular velocity which replaces an initial $\omega_{1}$ on a given site is independent of the sign of $\omega_{1}$.

Over a time scale during which recollisions could be neglected, the hard nature of the collision process leads us to expect that $F_{\omega}(t)$ decays exponentially. For long times, the mechanism that will alter the exponential decay is the probability of the return of $\omega_{1}$ to the initial site. Owing to the parity of the number of steps between the initial time and the return to its initial position, $\omega_{1}$ then has its original sign.

The central assumption of the theoretical considerations we shall make to understand the later numerical results is that the norms of the angular velocities (NAVS), which behave as quasi-particles, spread among the sites according to a normal diffusion law. We can then associate the diffusion of NAVS to a discrete random walk with step size and characteristic time adjusted to give the correct diffusion coefficient. The characteristic time $\tau_{0}$ of this random walk will generally be different from the mean collision time $\tau^{*}$ of the assembly of rotators. 100000 collisions were performed with an error estimated to be $0.3 \%$ for $F_{\omega}(t)$ for $t / \tau^{*} \leqslant 22.07$.

From the classical theory of diffusion processes, taking the lattice separation as unit length and $\tau_{0}$ as unit time scale, the mean square displacement of the NAVS for large time is

$$
\left\langle x^{2}(t)\right\rangle=2 D t
$$

with

$$
D=\left(\tau_{0} / \tau^{*}\right)^{2}
$$

As only the return of the NAVS to their initial site will alter the exponential decay of $F_{\omega}(t)$, this autocorrelation function will be given at long times by the null net displacement probability

$$
F_{\omega}(t) \simeq \frac{1}{2 \sqrt{\pi t}} \quad(\text { for } t \text { large enough })
$$

The results and method used here are, in our opinion, closely related to the most elementary approach of the long time tails of the linear velocity autocorrelation function in dimensions greater than one, as reported in the introduction of reference [5].

To check our theoretical considerations, we have carried out a molecular dynamic simulation; We considered 1000 rotators with an aperture angle of $\pi / 6$, with the interactions described above. We use the IBM 370-168 of CIRCE with double precision.

Table I gives $D$ deduced from the numerical results obtained separately on $\left\langle x^{2}(t)\right\rangle$ and $F_{\omega}(t)$. The numerical results confirm our theoretical conjecture.

Equation (3) is verified on figure 2, by a Ln-Ln plot of $F_{\omega}(t)$ versus $t$ which shows a straight line with slope $-1 / 2$. The theoretical prediction of the diffusive model is in good agreement with our numerical results up to more than twenty mean collision times. For very long times, we observe (Fig. 2) two seemingly erratic

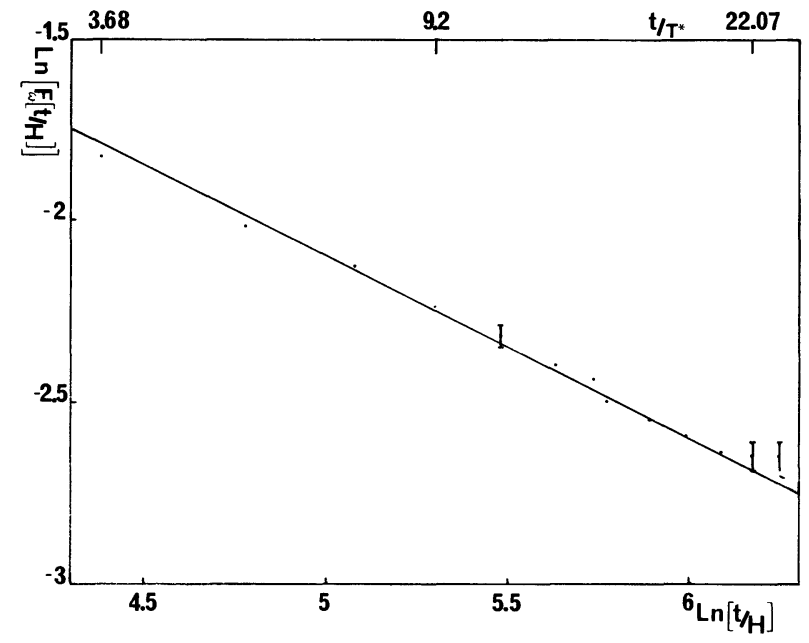

FIG. 2. - Ln-Ln plot of $F_{\omega}(t)$, denote experimental points. The solid line is a straight line with a slope of $-1 / 2 . H \simeq 4.6 \times 10^{-3}$ is an arbitrary unit of time used in the algorithm of integration.

results which might be due to statistical fluctuations or to the emergence of the artificial influence of the periodic boundary conditions.

Finally, note that the $t^{-1 / 2}$ decay is not universal for

\section{TABLE I}

\begin{tabular}{|c|c|c|c|c|c|}
\hline$t / \tau_{0}$ & $t / \tau^{*}$ & $F_{\omega}$ & $\left\langle x^{2}\right\rangle$ & $\begin{array}{l}D \text { calculated from } \\
\text { numerical results } \\
\text { on } F_{\omega}\end{array}$ & $\begin{array}{c}D \text { calculated from } \\
\text { numerical results } \\
\text { on }\left\langle x^{2}\right\rangle\end{array}$ \\
\hline- & - & - & - & - & - \\
\hline 6.99 & 9.2 & 0.107 & 12.5 & 0.86 & 0.89 \\
\hline 14.14 & 18.39 & 0.075 & 24.12 & 0.86 & 0.85 \\
\hline 15.54 & 20.23 & 0.072 & 26.46 & 0.85 & 0.85 \\
\hline
\end{tabular}

$D$ as calculated from the numerical results for $F_{\omega}$ and $\left\langle\Delta x^{2}\right\rangle$, using equations (1), (2) and (3) of the main text. Equation (3) is first used to compute $t / \tau_{0}$ from $F_{\omega}$ 
the angular velocity autocorrelation functions in one dimension : one can show, using the results of [4], that $F_{\omega}(t)$ has an exponential decay in a one-dimensional fluid of rough disks.
Acknowledgments. - This work had its origin in discussions with Pr. C. Brot. We would also like to thank Pr. J. Coste and Pr. J. Peyraud for valuable discussions and encouraging comments.

\section{References}

[1] Tresser, C., Quentrec, B. and Brot, C., J. Physique, 38 (1977) L-267;

Brot, C., Quentrec, B. and Tresser, C., Mol. Phys. 34 (1977) 1295.

[2] Mehaffey, J. R., Desai, R. C. and Kapral, R., J. Chem. Phys. 66 (1977) 1665 , and references quoted therein.
[3] BRot, C. and TRESSER, C., in preparation.

[4] JePSEN, D. W., J. Math. Phys. 6 (1965) 405.

Lebowitz, J. L. and Percus, J. K., Phys. Rev. 155 (1967) 122.

[5] Pomeau, Y., and Résibois, P., Phys. Rep. 19 (1975) 63, a review article dealing mainly with long time tail problems. 